\title{
$\begin{array}{ll}\text { Research Square } & \text { Preprints are preliminary reports that have not undergone peer review. } \\ \text { They should not be considered conclusive, used to inform clinical practice, }\end{array}$ or referenced by the media as validated information.
}

\section{A Novel RAC2 Mutation Causing Combined Immunodeficiency}

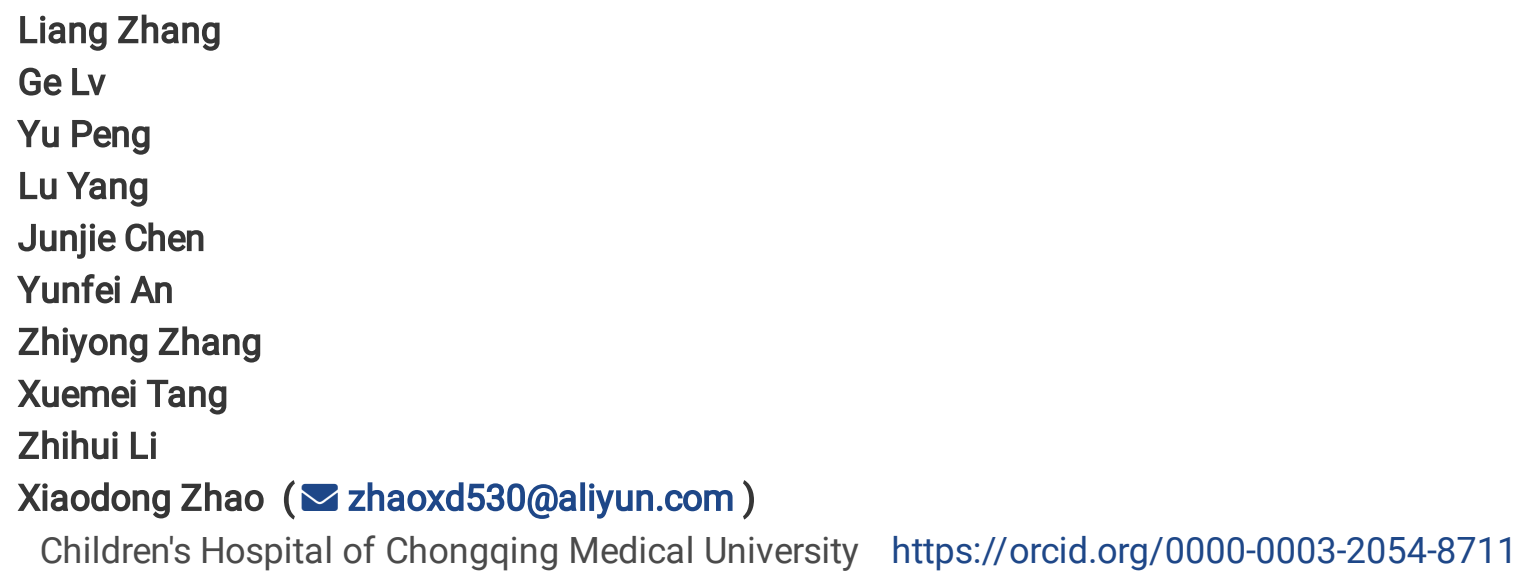

\section{Research Article}

Keywords: Ras-related C3 botulinum toxin substrate 2 (RAC2), combined immunodeficiency, gain of function mutation, penetrance

Posted Date: March 7th, 2022

DOI: https://doi.org/10.21203/rs.3.rs-1395115/v1

License: @ (i) This work is licensed under a Creative Commons Attribution 4.0 International License. Read Full License 


\section{Abstract \\ Purpose}

Ras-related C3 botulinum toxin substrate 2 (RAC2) acts as a molecular switch and has crucial roles in cell signaling and actin dynamics. A broad spectrum of genetic RAC2 mutations can cause various types of primary immunodeficiency, with complete penetrance. Here, we report a novel heterozygous missense mutation in RAC2 and the associated phenotypes in a Chinese family.

\section{Methods}

Immunological phenotype was detected by flow cytometry. T-cell receptor excision circles (TRECs) and K-deleting recombination excision circles (KRECs) were assessed by real-time quantitative PCR. Gene mutations were detected by whole-exome sequencing (WES) and confirmed by Sanger sequencing.

\section{Results}

The proband was an 11-year-old girl who presented with recurrent respiratory infections, bronchiectasis, persistent Epstein-Barr virus viremia, infectious mononucleosis, encephalitis, and cutaneous human papillomavirus infections. Laboratory analyses revealed increased serum IgG and decreased IgM levels, reduced naïve $\mathrm{CD} 4^{+}$and $\mathrm{CD} 8^{+} \mathrm{T}$ cells, an inverted $\mathrm{CD} 4^{+} / \mathrm{CD} 8^{+}$ratio, and low TREC and KREC numbers. WES identified a c.44G $>\mathrm{A}$ mutation in $R A C 2$ resulting in a p.G15D substitution. The proband's father suffered with recurrent respiratory infections and bronchiectasis, while her sister was apparently healthy, other than cutaneous human papillomavirus infections, despite both sharing the same mutation as the proband.

\section{Conclusions}

Our findings broaden the clinical and genetic spectra of $R A C 2$ mutations and underline the importance of $R A C 2$ gain-offunction mutations with complete or incomplete penetrance.

\section{Introduction}

RAC2 is a Rho guanosine triphosphatase (GTPase) which is exclusively expressed in hematopoietic cells and executes crucial physiological roles, including regulating hematopoietic stem cells, neutrophil migration and killing, and lymphocyte development and function, among others [1-3]. RAC2 can act as a molecular switch, which participates in gene transcription, cell survival, adhesion, reactive oxidant species (ROS) production, and cytoskeleton reorganization [4, 5]. Accordingly, variable mutations in $R A C 2$ have rarely been identified in a small number of patients, and result in different forms of primary immunodeficiency $[6,7]$.

The dominant negative monoallelic mutation of RAC2, p.D57N, predominantly causes defective neutrophil function, leading to recurrently progressive soft-tissue infections and wound healing failure [8-10]. By contrast, the recessive homozygous RAC2 mutation, p.W56X, causes common variable immunodeficiency, presenting as recurrent sinopulmonary infections and hypothyroidism, and characterized by severe B cell lymphopenia [11]. Further, the dominant activating monoallelic RAC2 mutations, p.G12R, p.P34H, p.Q61R, p.E62K, and p.N92T, cause severe combined immunodeficiency (SCID) or combined immunodeficiency (CID), with recurrent respiratory infections and bronchiectasis [12-17]. Overall, the available evidence demonstrates that different types of mutations in RAC2 are associated with vastly 
differently clinical phenotypes. Further, mutations causing RAC2 gain-of-function highlight the importance of tight regulation of the function of this molecule. All RAC2 mutations described to date cause immunodeficiencies with complete penetrance.

Here, we describe a novel heterozygous RAC2 missense mutation, c.44G >A (p.G15D), in an 11-year-old girl who presented with recurrent respiratory infections and bronchiectasis, cutaneous human papillomavirus infections, persistent EpsteinBarr virus (EBV) viremia, and EBV-induced disease, with low T-cell receptor excision circle (TREC) and K-deleting recombination excision circles (KREC) numbers, indicating immunodeficiency. We also report that the same mutation was shared with her father, who suffered with recurrent respiratory infections and bronchiectasis, and her sister who appeared healthy, apart from cutaneous human papillomavirus infections. Thus, our results suggest that dominant activating monoallelic RAC2 deficiency can exhibit incomplete penetrance.

\section{Materials And Methods}

\section{Ethics Approval and Consent}

This study was performed after obtaining written informed consent from the patient's guardians, consistent with the Declaration of Helsinki, and was approved by the Medical Ethics Committee of Hunan Children's Hospital.

\section{Genetic Studies}

Whole-exome sequencing (WES) was performed at Beijing Genome Institute (BGI, Shenzhen) on the Illumina Genome Analyzer platform. Genomic DNA was extracted from whole blood using a QIAamp DNA Mini Kit (Qiagen GmbH, Germany). Mutations in RAC2 were verified by Sanger sequencing using the following primer sequences: forward, 5囚TAGGCTGGGTGGATGCTGAG-3囚and reverse, 5囚-TCCTCCATACCCCATCCCGG-3囚.

\section{Flow Cytometric Evaluation of T and B Cells}

Peripheral lymphocyte phenotypes were quantified in separate experiments using $50 \mu$ whole blood samples, as previously described [18]. Samples were acquired on a FACSCanto II flow cytometer, and the data were analyzed using FlowJo software.

\section{Quantification of TRECs and KRECs}

Real-time quantitative PCR to detect TRECs and KRECs was performed as described previously [19].

\section{Studies of Oxidative Burst in Neutrophils}

Whole blood from patients and controls was stimulated with N-Formylmethionyl-leucyl-phenylalanine (fMLP) $(1 \mu \mathrm{M}$; Sigma-Aldrich), followed by dihydrorhodamine 123 staining.

\section{Results}

\section{Clinical Description}

The patient was from a nonconsanguineous kindred and had presented with recurrent respiratory infections since the age of 1 year, which occurred at a frequency of more than six times each year. She was repeatedly administered with oral and intravenous antibiotic treatment at local hospitals. She also experienced recurrent fever and petechia, along with an episode of convulsion at 1 year and 3 months old, so she was transferred to our hospital. At her first admission, laboratory findings indicated leukocytosis ( $14400 \mathrm{cells} / \mu \mathrm{L}$; normal range $4000-10000 \mathrm{cells} / \mu \mathrm{L}$ ), with $48.5 \%$ lymphocytes 
and $28 \%$ atypical lymphocytes, alongside anemia (hemoglobin, $77 \mathrm{~g} / \mathrm{L}$ ), thrombocytopenia (42000 cells $/ \mu \mathrm{L}$ ), and elevated liver enzymes (alanine transaminase $237.8 \mathrm{IU} / \mathrm{L}$; aspartate transaminase $564.7 \mathrm{IU} / \mathrm{L}$ ). Antiviral capsid antigen IgM to EBV was positive, and hepatosplenomegaly was detected on clinical examination and ultrasound. She was diagnosed with infectious mononucleosis and given intravenous ganciclovir treatment, along with antibiotic treatments for respiratory infection. After a month of hospitalization, leukocytes and thrombocytes returned to the normal range. She had undergone recurrent episodes of convulsion and fever at 4 years and 2 months old. EBV DNA was detected in the serum (1.31E+05 copies/mL) by viral DNA PCR (Table 1), along with transaminitis. Increased atypical lymphocytes with large vacuoles were observed by light microscopy (Fig. 1a). Cerebrospinal fluid (CSF) was colorless and not turbid, with normal cell counts and protein content, although glucose levels were elevated. Magnetic resonance imaging scan was performed and showed extensive and cytotoxic edematous changes in the left brain and thalamus (Fig. 1b, left). Based on these findings, viral encephalitis attributable to EBV was diagnosed. She received intravenous ganciclovir treatment. She has not had a convulsion episode since discharge; however, encephalomalacia, brain atrophy, and gliosis were detected in the left temporoparietal occipital lobe on re-examination 16 months later (Fig. 1b, right). Further, she experienced recurrent pneumonia along with episodes of asthma, and bronchiectasis was determined by chest computer tomography scan from 5 years old (Fig. 1c). Etiological examination verified repeated isolations of Haemophilus influenzae and Streptococcus pneumoniae from sputum samples. She has undergone repeated and prolonged hospitalization, as well as higher grade intravenous antibiotics treatment, approximately ten times annually.

Recently, primary immunodeficiency was suspected in the proband and she was admitted to our department at 11 years old. She was given interval intravenous immunoglobulin infusion, which significantly ameliorated her condition, and she has since required fewer and shorter hospitalizations.

The father of the proband is a 39-year-old man with a history of recurrent sinopulmonary infections and bronchiectasis, and has received traditional Chinese medicine treatment locally, while her sister is 9 years old and has cutaneous human papillomavirus infections, while appearing otherwise healthy, without recurrent infections.

\section{Immunological Assessment}

It was retrospectively noted that the proband's laboratory examinations showed an inverted CD4:CD8 ratio (0.43) and a reduced $\mathrm{B}$ cell percentage $(6 \%)$ at her first admission, as well as fluctuating neutropenia, progressive lymphopenia, and increased serum IgG levels, while IgM was decreased, except during her first episode of infectious mononucleosis (Fig. 1d and Table 2). Further investigations revealed that the patient had high relative percentages of effector/memory $\mathrm{CD} 4^{+}$and $\mathrm{CD}^{+} \mathrm{T}$ cells and transitional B cells, while CD4:CD8 ratio, naïve B cells, TRECs, and KRECs were markedly reduced, indicating immunodeficiency (Table 3). Neutrophils from the patient, her father, and her sister demonstrated increased oxidative burst capacity, relative to healthy control samples, after fMLP stimulation (Fig. 1e), consistent with the characteristic features of gain-of-function RAC2 mutations.

\section{Gene Sequencing Identified a Novel Heterozygous RAC2 Mutation}

In an attempt to define a genetic cause of the immunodeficiency, WES was performed and a previously unreported heterozygous variant, c. $44 \mathrm{G}>\mathrm{A}$, in $R A C 2$, was detected and subsequently confirmed by Sanger sequencing. This mutation was also detected in her father and younger sister by Sanger sequencing, while it was not found in her mother, uncle, or grandparents (Fig. 2a, b). This missense mutation results in a glycine to aspartic acid substitution at position 15 (p.G15D) of RAC2, which is predicted to be deleterious/pathogenic (Table 4). The missense mutated p.G15 residue is located within the G1 box (Fig. 2c), which is a highly conserved guanine nucleotide binding region [20]; therefore, the crystal structure of RAC2 was used as a template and the structural impact of the Gly15Asp mutant analyzed using Swiss PdbViewer. Structural analysis by 3D modeling showed that this mutation leads to destruction of hydrogen bonds and prevents interaction with GDP (Fig. 2d). According to the American College of Medical Genetics criteria [21], the p.G15D variant 
meets the pathogenic criteria: pathogenic moderate 1 (PM1), due to its location in a well-established functional domain; pathogenic moderate 2 (PM2), as the mutation is not reported in population databases, such as the 1000 Genomes Project; pathogenic supporting 1 (PP1), because the mutation cosegregates with disease in family members; and pathogenic supporting 3 (PP3), since multiple lines of computational evidence support a deleterious effect. Searches for RAC2 mutations in the COSMIC database of cancer genome mutations (Release V94;

http://cancer.sanger.ac.uk/cancergenome/projects/cosmic) revealed that somatic c.44G>A variants, leading to p.G15D amino acid substitution have been detected in human stomach adenocarcinoma. Together, these findings indicate that p.G15D is a plausible novel pathogenic heterozygous $R A C 2$ mutation.

\section{Discussion}

In this study, we identified a novel heterozygous RAC2 mutation, c.44G >A (p.G15D) in a Chinese family. Our findings broaden the genetic spectrum of RAC2 gain-of-function mutations and underlines that such mutations can cause immunodeficiency with either complete or incomplete penetrance.

Recent investigations of $R A C 2$ mutations have expanded understanding of the range of accompanying clinical presentations, which can manifest from the newborn period into adulthood. To date, RAC2 mutations have been reported in 18 patients with different forms of primary immunodeficiency. Recurrent respiratory infections leading to bronchiectasis development are shared phenotypes of patients with autosomal recessive loss-of-function RAC2 mutations and those with dominant gain-of-function mutations; these were also the main symptoms noted in our patient. Viral infections, including varicella zoster, herpes stomatitis, herpetic skin, shingles after varicella vaccination, and cutaneous human papillomavirus infections, have predominately been recorded in patients with RAC2 gain-of-function mutations [12-14]. Intriguingly, the proband in this study presented with persistent EBV viremia, infectious mononucleosis, and viral encephalitis, as well as cutaneous human papillomavirus infections.

EBV, also known as human herpesvirus 4, can cause severe disease in immunodeficient patients. EBV viremia and/or EBV-induced diseases, including fulminant infectious mononucleosis, hemophagocytic lymphohistiocytosis, lymphoproliferation, and lymphoma, are common clinical features of individuals affected with defined congenital primary immunodeficiencies. These immunodeficiencies primarily affect $\mathrm{CD} 8^{+} \mathrm{T}$ and NK cell functions, resulting in expansion or effector defects [22]. Examples of such immunodeficiencies include activated PI3Kס syndrome (APDS) [22, 23]. APDS is due to gain-of-function mutations in PI3KCD [24], and consequent direct binding and crosslinking between RAC and PI3K $[12,25]$; therefore, it has been assumed that many specific features and pathophysiological mechanisms are likely shared between patients with APDS patients and those with monoallelic activating RAC2 mutations [12, 26]. In this context, lymphopenia, with accumulation of senescent $\mathrm{CD} 8^{+} \mathrm{CD} 57^{+} \mathrm{T}$ cells and terminal effector $\mathrm{CD} 8^{+} \mathrm{T}$ cells, impaired T-cell receptor signaling, disturbed homing, and defective NK cell maturation or function, may theoretically lead to viral infections analogous to those characteristic of APDS, including with EBV, in patients with RAC2 mutations [12, 26]. This hypothesis is supported by evidence of increased Akt phosphorylation levels and modified degranulation in vitro following selective inhibition of p110 in patients with the RAC2 p.P34H mutation $[13,26]$.

It should be noted in our patient, that the involvement of neurological symptoms was accompanied by active EBV infection, characterized by elevated liver enzymes and atypical lymphocytes, and a particularly high serum EBV replication load. Despite the lack of serological confirmation and no detection of EBV DNA in CSF, EBV encephalitis was presumed. There was a previous case report of EBV-associated neurological involvement in primary immunodeficiency [27]. The amount of EBV DNA detected in serum correlates with her clinical symptom severity. Furthermore, cytotoxic edema can result in persistent disability, with irreversible brain tissue damage, which may indicate necrosis and unfavorable prognosis [28]. Our patient had cytotoxic edema, followed by atrophy, consistent with this scenario. Hence, 
we cannot rule out an alternative explanation that the lesions observed in our patient were caused primarily or only by EBV infection.

To our knowledge, the majority of patients with RAC2 mutations are characterized by reduced total lgG levels. Our patient was initially described as having clearly high IgG levels, resulting in reasonable suspicions of primary immunodeficiency disease being ignored. The mechanisms underlying this phenomenon remain unclear, although, possibly analogously, approximately $4 \%$ of patients with APDS also exhibit increased IgG levels [29]. Intriguingly, our patient only exhibited normal IgM levels at the onset of progressive infectious mononucleosis, then presented with constitutively decreased levels of this immunoglobulin, along with lymphopenia and undetectable serum EBV replication load. Further investigations into the unique role of RAC2 in humoral immunity and its distinct mutation spectrum are warranted.

Papilloma viral infections have been described in patients with the RAC2 p.P34H mutation [12], and were also observed in our patients with RAC2 p.G15D. This is reminiscent of homozygous nonsense RhoH mutations resulting in loss of RhoH protein, which led to T-cell defects causing susceptibility to papilloma virus infections in two adult siblings [30]. Mechanistically, RhoH is physiologically constitutively activated, and it has regulatory roles in competitive inhibition of Rac GTPases, coordinating as an antagonist at the downstream effectors of Rac [31]. Impairment of T-cell receptor function and reduced numbers of tissue homing integrin $\beta 7$-positive T cells likely contribute to susceptibility to $\beta$ papilloma viral infections in human $\mathrm{RHOH}$ deficiency [30]. Whether an analogical scenario underlies the effects of activating RAC2 mutations requires further study.

Finally, incomplete penetrance is frequently encountered in some forms of definitive primary immunodeficiency, predominantly those which are typically inherited in an autosomal dominant manner [32]. Hence, it is plausible that activating mutations in RAC2 can present with incomplete penetrance, similar to reports of APDS [31]. This hypothesis is further supported by the fact that the father of the proband in this study predominantly suffered with mild recurrent respiratory infections, while, by contrast, her younger sister appeared to be healthy, with no history of respiratory infections.

\section{Conclusion}

In conclusion, we report a Chinese patient with a novel heterozygous activating mutation in RAC2 who presented with combined immunodeficiency. Our findings broaden the clinical and genetic spectra of RAC2 mutations. Importantly, our results suggest that $R A C 2$ gain-of-function mutations can result in incompletely penetrant phenotypes.

\section{Declarations}

\section{Funding}

This work was supported by the Postgraduate Tutor Team Project of Chongqing Municipal Education Commission (Education Research and Development Program of Chongqing: 2019-9-66). L.Z. was also supported by the Talent Program of Hunan Children's Hospital (grant number: 1233).

\section{Competing Interests}

The authors declare no conflict of interest.

\section{Author Contributions}

ZX.D., and ZH.L. designed the study; L.Z., G.L., L.Y., and JJ.C. performed the experiments; Y.P. performed the genetic analysis; L.Z., and G.L. collected and analyzed the data, and wrote the manuscript; ZY.Z., YF.A., and XM.T. offered some 
crucial clinical data; all the authors reviewed the manuscript before it was submitted.

\section{Data Availability}

The data analyzed in the current study is available from the corresponding author on reasonable request.

\section{Ethics approval}

This study was approved by the Medical Ethics Committee of Hunan Children's Hospital (Approval number: HCHLL-202210).

\section{Consent to participate}

Informed consent was obtained from the patient and her parents, in accordance with the Declaration of Helsinki.

\section{Consent to publish}

Written informed consent was obtained from all participants included in the study.

\section{Acknowledgements}

We are grateful to the patient and her family for their continuous cooperation in this study. We thank the members of the laboratory for their technical assistance, and the doctors and nurses for their generous support of this project.

\section{References}

1. Bokoch GM. Regulation of innate immunity by Rho GTPases. Trends Cell Biol. 2005;15(3):163-71.

2. Williams DA, Zheng Y, Cancelas JA. Rho GTPases and regulation of hematopoietic stem cell localization. Methods Enzymol. 2008;439:365-93.

3. Pernis AB. Rho GTPase-mediated pathways in mature CD4+ T cells. Autoimmun Rev. 2009;8(3):199-203.

4. Heasman SJ, Ridley AJ. Mammalian Rho GTPases: new insights into their functions from in vivo studies. Nat Rev Mol Cell Biol. 2008;9(9):690-701.

5. Mulloy JC, Cancelas JA, Filippi MD, et al. Rho GTPases in hematopoiesis and hemopathies. Blood. 2010;115(5):93647.

6. Troeger A, Williams DA. Hematopoietic-specific Rho GTPases Rac2 and RhoH and human blood disorders. Exp Cell Res. 2013;319(15):2375-83.

7. Lougaris V, Baronio M, Gazzurelli L, et al. RAC2 and primary human immune deficiencies. J Leukoc Biol. 2020;108(2):687-696.

8. Ambruso DR, Knall C, Abell AN, et al. Human neutrophil immunodeficiency syndrome is associated with an inhibitory Rac2 mutation. Proc Natl Acad Sci U S A. 2000;97(9):4654-9.

9. Williams DA, Tao W, Yang F, et al. Dominant negative mutation of the hematopoietic specific Rho GTPase, Rac2, is associated with a human phagocyte immunodeficiency. Blood. 2000;96(5):1646-1654.

10. Accetta D, Syverson G, Bonacci B, et al. Human phagocyte defect caused by a Rac2 mutation detected by means of neonatal screening for T-cell lymphopenia. J Allergy Clin Immunol. 2011;127:535-538.

11. Alkhairy OK, Rezaei N, Graham RR, et al. RAC2 loss-of-function mutation in 2 siblings with characteristics of common variable immunodeficiency. J Allergy Clin Immunol. 2015;135(5): 1380-4.e1-5.

12. Lougaris V, Chou J, Beano A, et al. A monoallelic activating mutation in RAC2 resulting in a combined immunodeficiency. J Allergy Clin Immunol. 2019;143(4):1649-1653.e3. 
13. Hsu AP, Donkó A, Arrington ME, et al. Dominant activating RAC2 mutation with lymphopenia, immunodeficiency, and cytoskeletal defects. Blood. 2019;133(18): 1977-1988.

14. Sharapova SO, Haapaniemi E, Sakovich IS, et al. Heterozygous activating mutation in RAC2 causes infantile-onset combined immunodeficiency with susceptibility to viral infections. Clin Immunol. 2019;205:1-5.

15. Smits BM, Lelieveld PHC, Ververs FA, et al. A dominant activating RAC2 variant associated with immunodeficiency and pulmonary disease. Clin Immunol. 2020;212:108248.

16. Lagresle-Peyrou C, Olichon A, Sadek $\mathrm{H}$, et al. A gain-of- function RAC2 mutation is associated with bone-marrow hypoplasia and an autosomal dominant form of severe combined immunodeficiency. Haematologica. 2021;106(2):404-411.

17. Stern H, Donkó A, Shapiro T, et al. A Novel RAC2 Variant Presenting as Severe Combined Immunodeficiency. J Clin Immunol. 2021;41(2):473-476.

18. Ding Y, Zhou L, Xia Y, et al. Reference values for peripheral blood lymphocyte subsets of healthy children in China. J Allergy Clin Immunol. 2018;142(3):970-973.e8.

19. Sottini A, Serana F, Bertoli D, et al. Simultaneous quantification of T-cell receptor excision circles (TRECs) and Kdeleting recombination excision circles (KRECs) by real-time PCR. J Vis Exp. 2014;(94):52184.

20. Bunney TD, Opaleye O, Roe SM, et al. Structural Insights into Formation of an active signaling complex between Rac and phospholipase C Gamma 2. Mol Cell. 2009;34(2):223-33.

21. Richards S, Aziz N, Bale S, et al. ACMG Laboratory Quality Assurance Committee. Standards and guidelines for the interpretation of sequence variants: a joint consensus recommendation of the American College of Medical Genetics and Genomics and the Association for Molecular Pathology. Genet Med. 2015;17(5):405-24.

22. Latour S, Fischer A. Signaling pathways involved in the T-cell-mediated immunity against Epstein-Barr virus: Lessons from genetic diseases. Immunol Rev. 2019;291(1):174-189.

23. Cohen Jl. Herpesviruses in the Activated Phosphatidylinositol-3-Kinase- $\delta$ Syndrome.Front Immunol. 2018;9:237.

24. Lucas CL, Chandra A, Nejentsev S, et al. PI3Kס and primary immunodeficiencies. Nat Rev Immunol. 2016;16(11):702714.

25. Campa CC, Ciraolo E, Ghigo A, et al. Crossroads of PI3K and Rac pathways. Small GTPases. 2015;6(2):71-80.

26. Tabellini G, Baronio M, Patrizi O, et al. The RAC2-PI3K axis regulates human NK cell maturation and function. Clin Immunol. 2019;208:108257.

27. Weeks JK, Helton KJ, Conley ME, et al. Diffuse CNS vasculopathy with chronic Epstein-Barr virus infection in X-linked lymphoproliferative disease. AJNR Am J Neuroradiol. 2006;27(4):884-6.

28. Kim JH, Joo BE, Koh SB. Serial diffusion-weighted MR imaging findings in a patient with Epstein-Barr virus encephalitis. J Neurol. 2007;254(11):1616-8.

29. Coulter TI, Chandra A, Bacon CM, et al. Clinical spectrum and features of activated phosphoinositide 3-kinase $\delta$ syndrome:A large patient cohort study. J Allergy Clin Immunol. 2017;139(2):597-606.e4.

30. Crequer A, Troeger A, Patin E, et al. Human RHOH deficiency causes T cell defects and susceptibility to EV-HPV infections. J Clin Invest. 2012;122(9):3239-47.

31. Li X, Bu X, Lu B, et al. The hematopoiesis-specific GTP-binding protein RhoH is GTPase deficient and modulates activities of other Rho GTPases by an inhibitory function. Mol Cell Biol. 2002;22(4):1158-71.

32. Gruber C, Bogunovic D. Incomplete penetrance in primary immunodefciency: a skeleton in the closet. Hum Genet. 2020;139(6-7):745-757.

\section{Tables}


Table 1 EBV DNA copy numbers in the proband

\begin{tabular}{|llllll|}
\hline & $\begin{array}{l}\text { 4 years 2 } \\
\text { month old }\end{array}$ & $\begin{array}{l}\text { 4 years 3 } \\
\text { month old }\end{array}$ & $\begin{array}{l}\text { 8 years 8 } \\
\text { month old }\end{array}$ & $\begin{array}{l}\text { 11 years 7 } \\
\text { month old }\end{array}$ & Reference \\
\hline $\begin{array}{l}\text { Blood serum } \\
\text { (copies/mL) }\end{array}$ & $1.31 \mathrm{E}+05$ & $5.00 \mathrm{E}+03$ & $2.18 \mathrm{E}+04$ & 40.24 & $\begin{array}{l}\text { < lower detection } \\
\text { limit }\end{array}$ \\
\hline
\end{tabular}

Immunoglobulin levels in the proband without interval intravenous immunoglobulin infusion

\begin{tabular}{|llllll|}
\hline $\begin{array}{l}\text { Serum } \\
\text { Immunoglobulin }\end{array}$ & $\begin{array}{l}\text { 4 years } \mathbf{2} \text { month } \\
\text { old }\end{array}$ & $\begin{array}{l}\mathbf{5} \text { years } \mathbf{5} \text { month } \\
\text { old }\end{array}$ & $\begin{array}{l}\mathbf{8} \text { years } \mathbf{8} \text { month } \\
\text { old }\end{array}$ & $\begin{array}{l}\mathbf{1 0} \text { years } 7 \\
\text { month old }\end{array}$ & $\begin{array}{l}\mathbf{1 1} \text { years } 7 \\
\text { month old }\end{array}$ \\
\hline $\lg \mathbf{g}(\mathbf{g} / \mathrm{L})$ & $\begin{array}{l}14.10(6.60- \\
10.40)\end{array}$ & $\begin{array}{l}24.20(6.60- \\
10.40)\end{array}$ & $26.10(7.91-13.07)$ & $\begin{array}{l}29.50(7.91- \\
13.07)\end{array}$ & $\begin{array}{l}30.80(7.91- \\
13.07)\end{array}$ \\
\hline $\operatorname{lgA}(\mathbf{g} / \mathrm{L})$ & $2.52(0.58-1.00)$ & $1.52(0.58-1.00)$ & $3.89(0.85-1.71)$ & $4.13(0.85-1.71)$ & $4.32(0.85-1.71)$ \\
\hline $\operatorname{lgM}(\mathbf{g} / \mathrm{L})$ & $1.82(1.10-1.80)$ & $0.50(1.10-1.80)$ & $0.27(0.86-1.92)$ & $0.37(0.86-1.92)$ & $0.43(0.86-1.92)$ \\
\hline $\operatorname{lgE}(\mathbf{I U} / \mathbf{m L})$ & $271.10(<200.00)$ & $101.60(<200.00)$ & $1120.00(<200.00)$ & $244.00(<200.00)$ & $641.00(<200.00)$ \\
\hline
\end{tabular}

Table 3 Immunological profile of the proband 


\begin{tabular}{|c|c|c|c|c|}
\hline & Percentage & Reference range (\%) & Number/ $\mu l$ & Reference range (number/ $\mu \mathrm{L}$ ) \\
\hline T cells & 69.1 & $62.06-76.54$ & $925.5 \downarrow$ & $1297.20-2479.91$ \\
\hline $\mathrm{CD}^{+} \mathrm{T}$ cells & $48.7 \uparrow$ & $22.50-32.37$ & 651.9 & $508.71-1050.13$ \\
\hline CD8 ${ }^{+}$naïve & $10.9 \downarrow$ & $36.05-72.25$ & $71.1 \downarrow$ & $232.00-665.14$ \\
\hline CD $8^{+}$TEMRA & $33.4 \uparrow$ & $1.35-21.50$ & $217.7 \uparrow$ & $10.63-175.36$ \\
\hline $\mathrm{CD}^{+} \mathrm{CM}$ & $49.5 \uparrow$ & $13.05-39.45$ & $322.7 \uparrow$ & $99.72-300.57$ \\
\hline $\mathrm{CD}^{+} \mathrm{EM}$ & 6.3 & $2.00-16.75$ & 40.7 & $14.28-156.76$ \\
\hline $\mathrm{CD}^{+} \mathrm{T}$ cells & $19.4 \downarrow$ & $28.47-41.39$ & $260.4 \downarrow$ & $621.39-1258.00$ \\
\hline $\mathrm{CD4}^{+}$naïve & $26.6 \downarrow$ & $39.85-71.80$ & $69.3 \downarrow$ & $298.99-857.03$ \\
\hline $\mathrm{CD4}^{+}$TEMRA & 1.1 & $0.07-1.65$ & 2.8 & $0.60-14.51$ \\
\hline $\mathrm{CD}^{+} \mathrm{CM}$ & 46.0 & $23.25-51.30$ & $119.8 \downarrow$ & $218.53-463.39$ \\
\hline $\mathrm{CD}^{+} \mathrm{EM}$ & $26.4 \uparrow$ & $2.65-9.90$ & 68.7 & $24.20-94.14$ \\
\hline $\mathrm{TCRa}^{+} \mathrm{DNT}$ & 0.3 & $0.68-2.16$ & 2.3 & $11.79-41.44$ \\
\hline Yठ T & $1.9 \downarrow$ & $7.80-23.35$ & $17.1 \downarrow$ & $121.10-462.29$ \\
\hline B cells & 14.1 & $9.23-18.15$ & $188.4 \downarrow$ & $247.05-578.16$ \\
\hline Memory B & $4.2 \downarrow$ & $8.85-22.90$ & $7.8 \downarrow$ & $29.53-89.71$ \\
\hline Naïve B & $4.3 \downarrow$ & $44.95-75.80$ & $8.1 \downarrow$ & $140.30-380.88$ \\
\hline Transitional B & $34.2 \uparrow$ & $1.75-10.30$ & $64.4 \uparrow$ & $5.12-36.61$ \\
\hline Plasmablasts B & 2.6 & $0.70-7.95$ & 4.9 & $2.48-35.86$ \\
\hline NK cells & 16.8 & $7.75-23.47$ & 225.7 & $202.54-583.53$ \\
\hline CD4:CD8 ratio & $0.4 \downarrow$ & & & \\
\hline TREC & $5 \downarrow$ & $6 \downarrow$ & & \\
\hline KREC & $22 \downarrow$ & $7 \downarrow$ & & \\
\hline
\end{tabular}

TEMRA, terminally differentiated effector memory helper T lymphocytes; CM, central memory; EM, effector memory.

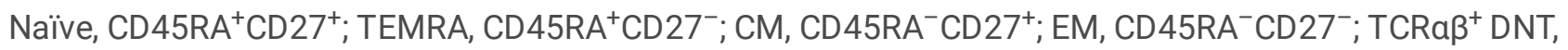
$\mathrm{CD}^{+}{ }^{+} \mathrm{CRa} \beta^{+} \mathrm{CD} 4^{-} \mathrm{CD} 8^{-}$; Memory B cells, $\mathrm{CD} 19^{+} \mathrm{CD} 27^{+} \mathrm{IgD}{ }^{-}$; naïve B cells, $\mathrm{CD} 19^{+} \mathrm{CD}_{27^{-}} \mathrm{IgD}^{+}$; transitional $\mathrm{B}$ cells, $\mathrm{CD} 19^{+} \mathrm{CD} 24^{++} \mathrm{CD} 38^{++}$; plasmablasts, CD19+CD24-x0005-CD38 ${ }^{++}$. KRECs, kappa-deleting recombination excision circles; TRECs, T-cell receptor excision circles.

Table 4 Prediction of $R A C 2$ mutation pathogenicity 


\begin{tabular}{|cllllll|}
\hline Variant & Provean & Provean & MutationTaster & MutationTaster & PolyPhen-2 & PolyPhen-2 \\
& Score & Prediction & Prediction & Prob & HumDiv & HumVar \\
\hline G15D & -6.16 & Deleterious & Disease causing & 1.00 & 0.97 & 0.95 \\
\hline
\end{tabular}

\section{Figures}

\section{Figure 1}

Clinical features of the patient. (a) Large vacuoles (arrows) were detected by light microscopy in neutrophils isolated from bone marrow when the patient was aged 3 years and 4 months. (b) Magnetic resonance imaging (MRI) scans of the patient at age 3 years and 4 months. (c) Computed tomography (CT) scans of the patient at age 5 years. (d) Absolute counts of white blood cells, neutrophils, and lymphocytes over time. (e) Oxidative burst in dihydrorhodamine 123-loaded neutrophils from patients P1 (the proband's father), P2 (the proband), P3 (younger sister), and the proband's mother after stimulation with fMLP

\section{Figure 2}

A novel RAC2 mutation identified within the highly conserved G1 box. (a) The family pedigree. (b) Results of Sanger sequencing of $R A C 2$ exon 2 demonstrating the presence of the c.44G>A variant in patients $\mathrm{P} 1-\mathrm{P} 3$ and wild-type sequence in the proband's mother. (c) Schematic representation of the RAC2 protein and its mutations (upper), and amino acid alignment of the highly conserved G1 box across different species (lower). All mutations identified to date are indicated in the protein domains, with reported dominant activating monoallelic mutations depicted as red vertical bars. (d) Structural analysis of the mutant RAC2 protein. Three-dimensional models of the RAC2 p.G15D mutant are shown with effects on GDP binding. Computer predicted hydrogen bonds are shown as green dashed lines 